\title{
ICE PACK TEST-AN ALTERNATE TO PROSTIGMIN TEST IN THE DIAGNOSIS OF MYASTHENIA GRAVIS WITH OCULAR INVOLVEMENT: A SIMPLE, NONINVASIVE AND SAFE METHOD
}

\author{
SHARIF AQMO ${ }^{1}$, FARUQUE GM $^{2}$, ANISUDDIN AIM ${ }^{3}$, KABIR MF $^{4}$, ZAFARULLAH TA $^{5}$
}

\begin{abstract}
:
Myasthenia gravis (MG) is the most frequent neuromuscular transmission disorder with incidence of 2-20 patients per million ${ }^{1}$. Its pathophysiology is autoimmune, with acetylcholine receptors (AChR) autoantibodies damaging the post-synaptic fold at the muscle membrane. MG often presents with isolated ocular symptoms, including diplopia and ptosis, at least at the onset. It is often challenging to confirm the diagnosis of MG. The most traditional diagnostic test for myasthenia gravis is the edrophonium / prostigmin test. However, with this test, false-positive results have been reported in other neurologic disorders like amyotrophic lateral sclerosis, botulism and brain tumour. Furthermore, it also carries a risk of serious cardiac adverse reactions. The ice pack test is a very simple, safe and cheap procedure that can be performed by the physician at the bedside. Considering that the pure ocular form of $M G$ is frequently not detected by the traditional tests available, the ice pack test is an attractive diagnostic method. We describe two cases where the ice pack test was done at the bedside for the diagnosis of MG and then performed prostigmin test and reviewed the literature about this test.

In conclusion, the ice pack test is an easy, safe, cheap and reliable test to be used at bedside in the MG suspected patients with ptosis. It is safer and can replace the edrophonium/ prostigmin test in MG with ptosis, since the specificity and sensitivity is 100\% when compared.
\end{abstract}

Key words: Myasthenia Gravis (MG), prostigmin test, occular menifestation.

J Dhaka Med Coll. 2009; 18(2) : 112-114

\section{Introduction:}

Myasthenia gravis (MG) is the most frequent neuromuscular transmission disorder with incidence of 2-20 patients per million ${ }^{1}$. Its pathophysiology is autoimmune, with acetylcholine receptors (AChR) autoantibodies damaging the post-synaptic fold at the muscle membrane. MG often presents with isolated ocular symptoms, including diplopia and ptosis, at least at the onset. In up to $15 \%$ of the patients the disease remains confined to the eyes. Other clinical features include bulbar symptoms (dysphagia, dysarthria), proximal limb muscles weakness and fatigue and even distal limb muscles weakness ${ }^{2,3}$. The main characteristic of the MG is the fluctuating weakness during the day, being milder during the morning and more severe at the evening. The weakness usually worsens with physical activity. It is often challenging to confirm the diagnosis of MG. The tests usually performed to confirm the diagnosis are the edrophonium test (Tensilon $\left.{ }^{\circledR}\right)$ /Prositgmin test, repetitive nerve stimulation (RNS), single fiber EMG and serum acetilcholine receptors (AchR) antibodies.The later three being unavailable in our country. The most traditional diagnostic test for myasthenia gravis is the edrophonium / prostigmin test. However, with this test, false-positive results have been reported in other neurologic disorders like amyotrophic lateral sclerosis, botulism and brain tumor. Furthermore, it also carries a risk of serious cardiac adverse reactions. In pure ocular disease its sensitivity

1. Registrar, Dept. of Ophthalmology, Dhaka Medical College Hospital, Dhaka.

2. Asst. Professor, Dept. of Ophthalmology, Dhaka Medical College \& Hospital, Dhaka.

3. Asst. Professor, Dept. of Ophthalmology, Sir Salimullah Medical College \& Hospital, Dhaka.

4. Associate Professor. Dept. of Ophthalmology, Chittagong Medical College \& Hospital, Chittagong.

5. DO (DU)

Correspondence: Dr. AQM Omar Sharif. 
is about $86 \% .{ }^{4}$ Another bedside test that can be done is the sleep test, however this test is time consuming and probably not practical for the busy physician. The other complementary tests have variable sensitivity and specificity. The AChR antibodies are the most specific test for myasthenia gravis, however the sensitivity varies from $56 \%$ in pure ocular myasthenia according to Tabassi et al. ${ }^{5}$ to $70 \%$ in Oh et a 1 . ${ }^{6}$ study.. The most sensitive test for ocular myasthenia is single fiber EMG, reaching up to $80 \%$ in pure ocular cases and $95 \%$ in generalized disease, ${ }^{6}$ but its specificity is low, the test is expensive, technically demanding and not available in our country..

The ice pack test is a very simple, safe and cheap procedure that can be performed by the physician at the bedside. ${ }^{7}$ Moreover, the ice pack test does not require medications or expensive equipment and is free of adverse effects ${ }^{8}$. It consists of the application of an ice pack on the patient symptomatic eye for 3 to 5 minutes. The response is positive when there is improvement of the diplopia or ptosis (increase in at least $2 \mathrm{~mm}$ of the palpebral fissure from before to after the test). Considering that the pure ocular form of MG is frequently not detected by the traditional tests available, the ice pack test is an attractive diagnostic method. We describe two cases where the ice pack test was done at the bedside for the diagnosis of MG and then performed prostigmin test and reviewed the literature about this test. The patient have signed an informed consent authorizing the publication of the cases .

\section{Cases :}

\section{Case I}

A 27 year old male came to our hospital with drooping of the upper eye lid for 23 days in the right eye. The drooping typically worsens as the day progresses and on physical exertion. . The neurologic examination showed only left eye ptosis of about $3.5 \mathrm{~mm}$ and no ophthalmoparesis. The ice pack test was then performed to confirm the diagnosis (residual ptosis being $0.5 \mathrm{~mm}$ ). The next day
Edrophonium test was performed to confirm and compare. The test was positive.

Case 2:

A 31 year old female came to Dhaka medical college hospital with drooping of upper eye lids and double vision (diplopia) for 12 days.Neurological examination showed bilateral ptosis of $2 \mathrm{~mm}$ with ophthalmoparesis in the right eye. Ice pack test was positive .

\section{Discussion :}

Clinical and laboratorial experiences showed an important correlation between temperature and myasthenia gravis 12 . It is widely known that warm temperatures worsen MG symptoms and cooling is able to ameliorate them ${ }^{9-11}$. The improvement of MG with cooling probably occurs by lesser acetylcholinesterase activity in temperatures below $28^{\circ} \mathrm{C}$, providing increasing amount of $\mathrm{ACh}$ molecules in the synaptic cleft ${ }^{12}$. Based on this evidence, orbital cooling (ice pack test) has been developed as a simple, safe and reliable procedure for the diagnosis of ocular myasthenia ${ }^{13,14}$.

The ice pack test seems to be both sensitive and specific when compared with the prostigmin test ${ }^{15}$ although there are no randomized and controlled studies to validate it. The sensitivity in ocular myasthenia with ptosis associated or not with generalized symptoms is high varying from 80 to $100 \% 7,15$. The specificity of the ice pack test is very high considering that it is not positive in other diseases that can simulate MG such as oculomotor nerve palsy, oculopharyngeal muscular dystrophy and mitochondrial myopathies. False-positive results were virtually not seen $7,15,16,18,19$. In a large study with 156 patients comparing ice pack test with the edrophonium test, the ice test had sensitivity and specificity of $100 \%{ }^{5}$. In such study, all cases with positive edrophonium test had positive ice test whereas none of the cases with negative edrophonium test had positive ice test. Therefore, the confidence of the test in the diagnosis of the MG is very good, supporting its use in the diagnosis of $\mathrm{MG}^{17}$.In our test we used prostigmin in case of edrophonium.

Although cooling is able to improve the muscle strength in myasthenic patients, decrease the muscle temperature below $22^{\circ} \mathrm{C}$ is not recommended because it can cause decrease 
the muscle fiber contraction force, rather than improve the neuromuscular transmission ${ }^{20}$.

The reliability of the ice test in patients with mild ophthalmoplegia and diplopia without ptosis is probably lesser, because the findings are subjective. Saavedra et al. ${ }^{14}$ have not found any definite effect on ocular movements after the ice pack test, although Larner and Thomasl stated clear subjective and objective effect of the ice pack test on eye movements. Except for this finding, further investigations and treatment provided no other evidence of MG in their case, making diagnosis uncertain. They recommended caution in making $\mathrm{MG}$ diagnosis based only on the ice pack test for patients with isolated diplopia and mild ophthalmoparesis, without any ptosisl.

Benatar $^{21}$ highlights that the majority of the literature studies about ice pack test in MG has employed a case-control design, in which patients with previous diagnosis of $M G$ were compared to a control group. Usually these studies have a tendency to overestimate both sensitivity and specificity ${ }^{22}$. These methodological limitations need to be considered during the ice test interpretation.

\section{Conclusion :}

In conclusion, the ice pack test is an easy, safe, cheap and reliable test to be used at bedside in the MG suspected patients with ptosis. It is safer and can replace the edrophonium/ prostigmin test in MG with ptosis, since the specificity and sensitivity is $100 \%$ when compared $^{5}$. It is useful even in patients with negative serum AChR antibodies. Caution should be taken on the interpretation of the ice test in patients with isolated diplopia without ptosis, mainly when there is no definite ophthalmoplegia. The major disadvantage of the ice test is that it is only applicable when ptosis is present ${ }^{21}$. Thus it is not helpful in patients with isolated proximal limb weakness.A further multicenter study is required for better evaluation.

\section{References:}

1. Larner AJ, Thomas DJ. Can myasthenia gravis be diagnosed with the ice pack test? A cautionary note. Postgrad Med J 2000;76:162-163.

2. Scola RH, Iwamoto FM, Annaai M, et al. Miastenia grave distal. Arq Neurop Psiquiatr, 2003;6L119120.
3. Oh SJ. Cho HK. Edrophonium responsiveness not necessarily diagnostic of myasthenia gravis. Muscle Nerve 1990;13:187-191.

4. Philllips LH, Melnick PA. Diagnosis of myasthenia gravis in the 1990s. Semin Neurol 1990;10:62-90.

5. Tabassi A, Dehghani A, Saberi B. The ice test for diagnosing myasthenia gravis. Acta Medica Iranica 2005;43:60-62.

6. Oh SJ, Kim DE, Kuruoglu R, Bradley RJ, Dwyer D. Diagnostic sensitivity of the laboratory tests in myasthenia gravis. Muscle Nerve 1992;15:720-724.

7. Lertchavanakul A, Gamnerisiri P, Hirunwiwatkul P. Ice test for ocular myasthenia gravis. J Med Thai 2001;84(Suppl 1):S131-S136.

8. Larner AJ. The place of the ice pack test in the diagnosis of myasthenia gravis. Int $\mathrm{J}$ Clin Pract 2004;58:887-888.

9. Gutmann L. Heat exacerbation of myasthenia gravis. Neurology 1978; 28:398. 10. Gutmann L. Heat-induced myasthenia crisis. Arch Neurol 1980;37:671-672.

11. Ricker K, Gertel G, Stodieck S. Influence of temperature on neuromuscular transmission in myasthenia gravis. J Neurol 1977;216:273-282.

12. Simpson JA. Myasthenia gravis: a new hypothesis. Scott Med J 1960; 5:419-436.

13. Borestein S, Desmedt JC. Local cooling in myasthenia. Improvement of neuromuscular failure. Arch Neurol 1975;32:152-157.

14. Saavedra JS, Femminini R, Kochen S, Ortiz de Zarate JC. A cold test for myasthenia gravis. Neurology 1979;29:1075.

15. Santos-Lasaosa S, Pascual-Millan LF, JericoPascual I, Larrode-Pellicer P. Aplicacion del test del hielo en el diagnostico de la miastenia grave. Rev Neurol 2002;34:299-300.

16. Golnik KC, Pena R, Lee AG, Eggenberger ER. An ice test for the diagnosis of myasthenia gravis. Ophthalmology 1999;106:1282-1286.

17. Czaplinski A, Steck AJ, Fuhr P. Ice pack test for myasthenia gravis: a simple, noninvasive and safe diagnostic method. J Neurol 2003;250:883-884.

18. Movaghar M, Slavin ML. Effect of local heat versus ice on blepharoptosis resulting from ocular myasthenia. Ophathalmology 2000; 107: 22092214.

19. Kubis KC, Danesh-Meyer HV, Savino PJ, Sergott $\mathrm{RC}$. The ice test versus the rest test in myasthenia gravis. Ophthalmology 2000;107:1995-1998.

20. Benatar M. A systematic review of diagnostic studies in myasthenia gravis. Neuromuscular Disord 2006;16:459-467.

21. Lijmer JG, Mol BW, Heisterkamp S, et al. Empirical evidence of design-related bias in studies of diagnostic tests. J Am Med Assoc 1999;282: 1061-1066. 